\title{
DEEP NECK INFECTIONS - COMPLICATIONS AND SURGICAL TERATMENT
}

\author{
Kirov G, Benchev R., Stoyanov St., Kondarev M. \\ Medical Institute of the Ministry of Interior, Sofia \\ Reviewed by: Assoc. prof. R. Madjov, PhD
}

\begin{abstract}
Introduction: Deep infections of the neck are potentially life-threatening due to their descending spread towards the mediastinum along cervical fascia planes and possible development of thrombophlebitis of the internal jugular vein and sepsis. Aim: To review the possible complications of the deep neck infections and the methods for surgical treatment. To share our own clinical experience. Material: 29 patients with deep neck infections predominantly of tonsillar or dental origin, treated surgically at the Medical Institute of Ministry of the Interior during the last 5 years. Results: The following complications have been observed: 6 cases of acute respiratory insufficiency; 2 cases of sepsis; 2 - with descending mediastinitis and 1 - with acute hemorrhage from gastric stress ulcer. Conclusions: Early surgical intervention, application of antibiotics against aerobe and anaerobe bacteria and good coordination between otorinolaryngologists, surgeons, ICU and microbiologists is essential for the successful treatment of deep neck infection.
\end{abstract}

Keywords: deep neck infections, surgery, mediastinitis, abscess, phlegmona.

\section{INTRODUCTION}

As deep neck infections are defined the infections which arise in or spread to the deep cervical compartments. Deep neck abscesses and phlegmonas are usually result of inadequately treated tonsillitis, but dental origin is also possible. After incision and application of a suitable antibiotic, they are usually cured without additional complications. Sometimes, in cases of heavier clinical course, wrong treatment or severely impaired immune system, development of life threatening complications as descending mediastinitis and sepsis, is possible.

In the present paper, we are reviewing some complications of deep neck infections and their surgical treatment at the MI-MI during the last 5 years.

\section{MATERIAL AND METHODS}

For the period 1999-2003 have been treated 29 patients with deep cervical abscesses or phlegmonas. In 12 patients was found submandibular abscess; 10 were with lateropharyngeal abscess; 3 with angina Ludwici; 3 with neck phlegmonas and 1 with cervical exteriorization of mastoiditis. 2 patients later developed mediastinitis and 2 sepsis. 6 patients underwent tracheostomy for acute respira-

Address for correspondence:

Kirov G, Medical Institute of the Ministry of Interior, Sofia, BULGARIA tory insufficiency. One patient had massive hemorrhage from gastric stress ulcer. Microbiological investigation found St. aureus in 30\% of the cases, beta-hemolytic streptococcus in $25 \%$, Proteus and Pseudomonas aerug. in 25\%, Str. Pneumoniae in $10 \%$ and anaerobes in $10 \%$.

Surgical treatment consisted of cervical incisions and draining of the affected areas in cases with deep neck infections and cervicomediastinal draining or thoracotomy in cases with descending mediastinitis. Simultaneously was carried out intensive parenteral antibiotic treatment, in accordance with the results from the antimicrobial susceptibility testing. Surgical incisions were left open wide for better aeration and cleaning of necrotic materials.

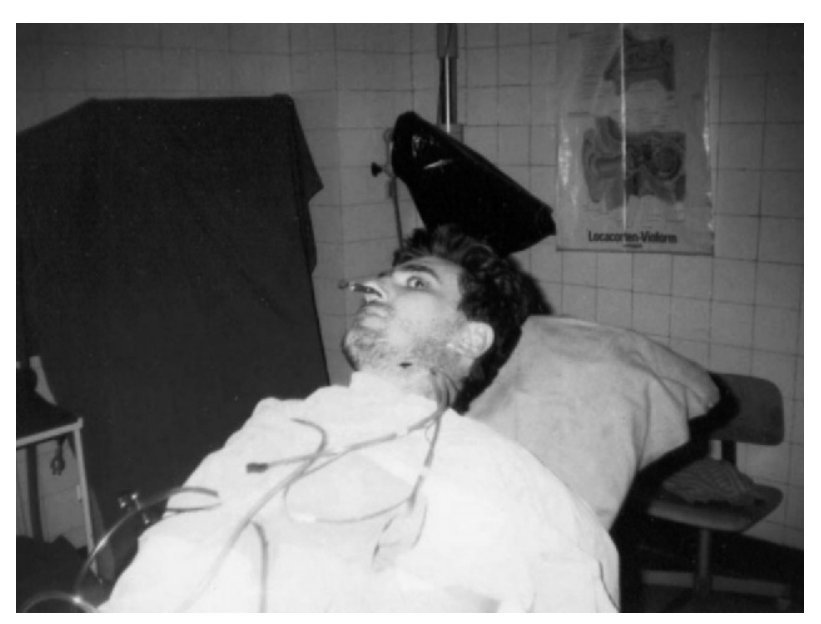

Fig. 1 . 
Kirov G, Benchev R., Stoyanov St. ...

The draining of deep cervical and mediastinal spaces was accomplished by a system for continuous aspiration and wash out, consisting of catheters No 18 or 20, connected to the system for central aspiration and thinner catheters for wash out of the abscess cavities (fig.1). During the first few days after the intervention, the aspiration was continuous, but later after improvement of the general and local state it was done in intervals of several hours.

Together with the surgical treatment was carried out intensive antibiotic therapy. Usually we started with Amoxicillin with beta-lactamase inhibitor and Metronidazol and later proceeded in accordance with the antibiogram results. Often we applied the combination Clindamycin and Amikacin for about 10 days and continued with 3rd generation cephalosporins or quinolones. For prophylaxis of the edema and respiratory insufficiency we used Methilprednisolon, but in 6 cases tracheostomy was necessary. In patients with disphagia was placed nasogastric feeding tube in order to oppose protein deficits and additional weight loss.

Treatment of deep neck infections usually lasted 2 - 3 weeks, and in cases with sepsis and mediastinitis - about 5 6 weeks.

We present 3 cases with deep neck infections, which according to us are interesting because of the severe and unusual complications.

\section{CASE 1}

37 years old male, admitted in a state of emergency, with severe pulsating headache, profuse ear suppuration, fever, reaching $39 \mathrm{oC}$, painful swelling at the right side of the neck, paralysis of the right facial half, and difficult head movement. The complaints date back from 3 weeks, but the parotid swelling appeared 10 days ago and gradually increased. The patient did not seek medical care, but conducted selftreatment with analgetics and vitamins. Systematically abused with alcohol.

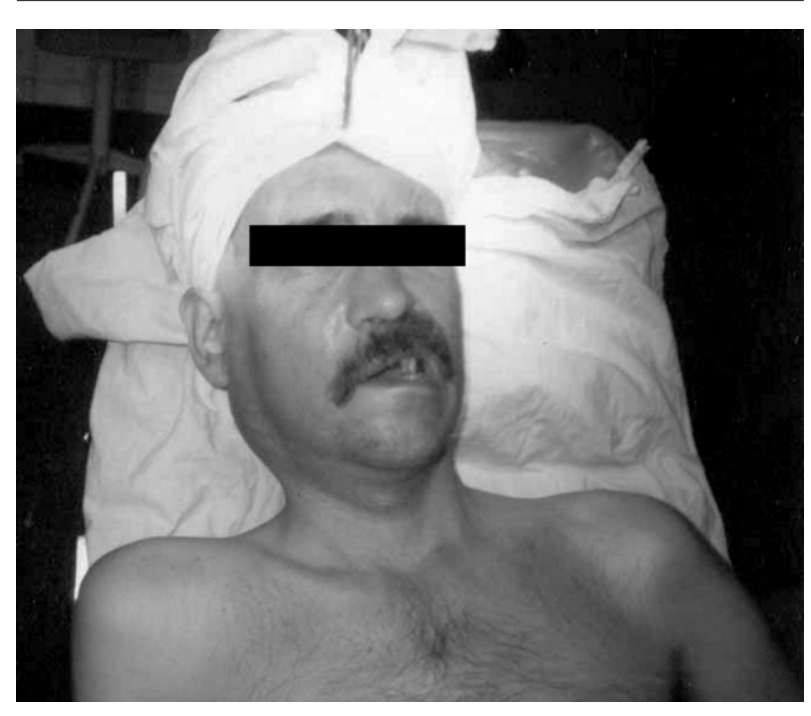

Fig. 2.
At the admission, the patient was in impaired general condition, adequate, febrile, RR 120/80 mm Hg, heart rate 85 beats / min and permanent purulent discharge from the right ear. The back and the right half of the neck were swollen and painful with skin hyperemia. The edema reached $5 \mathrm{~cm}$ above the clavicle and when pressed provoked increase of the ear suppuration (symptom of Luc). The right shoulder stood lower and the abduction of the right arm was difficult. The head was tilted to the right. The right facial half was with decreased mobility (peripheral paresis) (fig. 2). The $\mathrm{x}$-ray of proc. mastoideus was without signs of mastoiditis. The audiogram showed conductive hearing loss. Laboratory tests revealed: leukocytosis $(15,2 \mathrm{~g} / \mathrm{l})$ and ESR 110/130 $\mathrm{mm}$. Microbiology showed St. aureus and anaerobes.

Urgent operation was performed. Under general anesthesia was done mastoidectomy with amputation of the mastoid apex, incision and draining of the cervical phlegmona. The mastoid cavity was full with purulent secretion and easy bleeding granulations. No dehiscence or erosions of the facial canal were found. The pus passed through the mastoid apex under $\mathrm{m}$. sternocleidomastoideus - exteriorization in the areas of Bezold and Mouret. The pus collection spread medially and posteriorly to the scullbase and the vertebra and caudally along the neurovascular bundle in front and behind the cervical spine. Through an incision at the front edge of $\mathrm{m}$. sternocleidomastoideus were evacuated about $250 \mathrm{ml}$ of pus. Draining tubes for continuous aspiration and wash out were placed. The wound was left open for better aeration and cleaning.

Antibiotic therapy with Amoxicillin - clavulanate, Amikacin and Metronidazol was started. The general state of the patient gradually improved. After 10 days, the treatment continued for another 7 days with Lincomycin, and later - Septrin. The drains were removed sequentially. After eradication of the infection the wound was sutured. The paresis of CSN VII and XI subsided and hearing returned to normal. The patient was dismissed in good general condition after 43 days.

\section{CASE 2}

59 years old female, admitted with left peritonsillar abscess and cervical lymphadenitis. The abscess was incised and therapy with Amoxicillin started. The next day body temperature raised to $40 \mathrm{oC}$. Hypopharyngeal edema, pain and neck swelling appeared. Surgical drainage of the left cervical region was done, and lateropharyngeal abscess was found. About 100ml of pus were aspirated. Aspiration and wash out catheters were placed. The microbiological investigation showed beta hemolytic streptococcus group $\mathrm{A}$. The dose of Amoxicillin was increased. Amikacin was added to the therapy. In spite of the treatment, the infection spread caudally and the patient developed descending mediastinitis. The initial incision in front of $\mathrm{m}$. sternocleidomastoideus was enlarged to the jugulum and jugulotomy was performed. A massive pus collection in the anterosuperior mediastinum was drained. 3 catheters were 
placed in the mediastinum - 2 for aspiration and 1 for irrigation. The incision was left open for better aeration. The hemoculture showed sepsis, caused by Enterobacter. The treatment continued with Clindamycin and Amikacin for 10 days, and Ceftazidim with Metronidazol for another 12 days and finished with Ciprofloxacin. Three blood transfusions were made because of anemia and leukocytopenia. Gradually the condition of the patient improved, the drains were removed and the wound closed. She was dismissed after 35 days.

\section{CASE 3}

51 years old male, admitted in impaired general state, with fever, difficult and painful swallowing, drooling, neck edema and trismus. About a week ago was carried out difficult tooth extraction for dental infection. He was treated with Amoxicillin and Gentamycin, but the infection spread to the submental area and later bilaterally to the deep cervical compartments. At the admission, the floor of the oral cavity, hypopharynx and larynx were edematous. Saliva as leaking to the larynx and caused cough and dispnea.

Urgent surgical treatment was done. The neck was drained through several incisions, and the affected areas were open bilaterally. For maintenance of the respiratory ways and aspiration of the secretions was performed tracheostomy (fig.3).

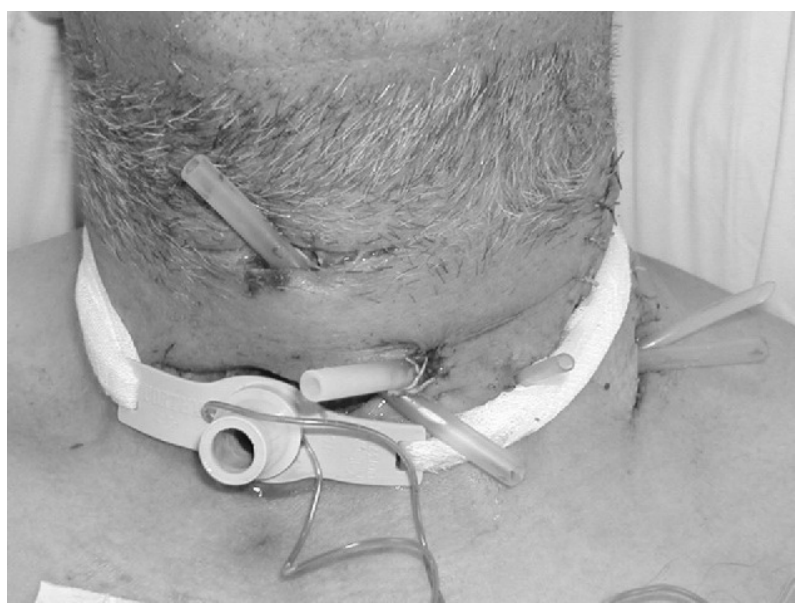

Fig. 3.

Nasogastal tube was placed with some difficulty due to the hypopharyngeal edema. Therapy with Cephtriaxon, Metronidazol, Methylprednisolon, vitamins, serum and glucose infusions was started. At the third day after the hospitalization, the patient had melena. Fibrogastroscopic examination was impossible because of hypopharyngeal edema. As bleeding continued, laparotomy was performed. Several gastric ulcers were found and were sutured. Later the patient had ileus, which implicated relaparotomy with formation of laparo- and ileostoma (fig.4). Due to the impaired general state he developed sepsis. Microbiological investigation showed Pseudomonas aeroginosa, suscepti- ble only to Imipenem. The treatment was changed in accor-

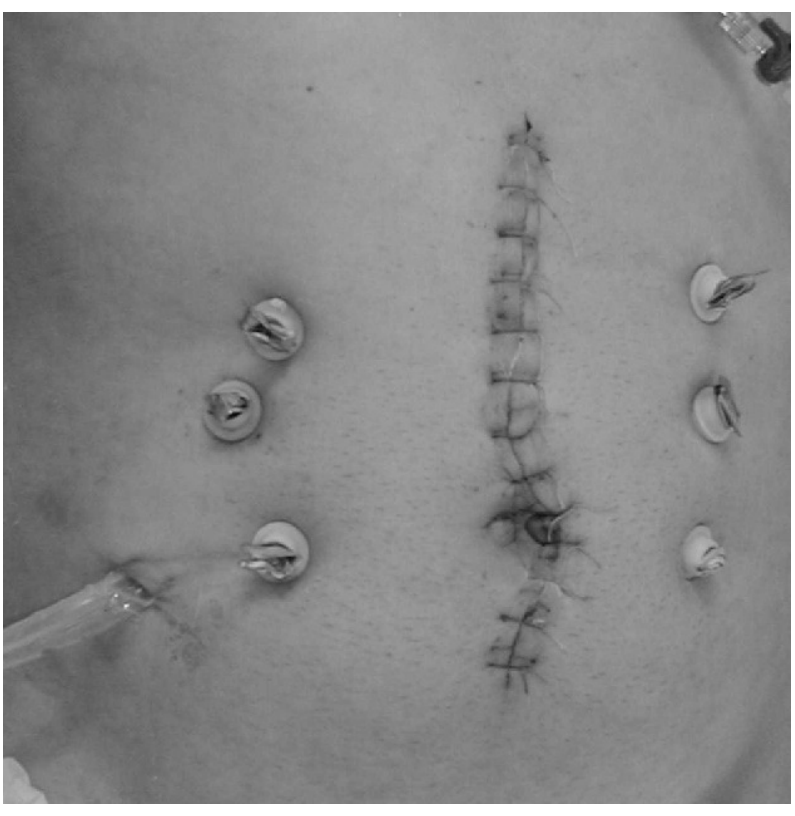

Fig. 4.

dance with the antibiogram.

Gradually the state improved. The neck infection was treated. The drains and the nasogastric tube were removed. The patient was decanulated. About a week later dispnea appeared again. Endoscopically was diagnosed tracheal stenosis at the level of the tracheostomy opening. The patient was retracheotomized and the granulations were excised with APC. Two weeks later the he was decanulated. A month later was done successful restitution of the ileum.

\section{RESULTS AND DISCUSSION}

All patients with one exception recovered after the treatment. One patient with neck phlegmonas died from cardiac arrest in the operating theatre after urgent intubation and tracheostomy for acute respiratory failure.

Before the hospitalization, most patients with deep abscesses or phlegmonas had been treated with low antibiotic doses. The others had not been consulted by specialist before the complications developed. There were 2 cases, in which, in spite of the early incision and parenteral antibiotic treatment, the infection spread to the neck and mediastinum.

Anatomical predispositions for the development of deep neck infections are related to the topography of the deep cervical fascia, which forms planes and spaces in the neck, containing fat tissue, blood and lymphatic vessels. The infection of these spaces leads to development of deep abscesses and phlegmonas. When the pressure of the pus collection rises, the process may spread along the fascial planes in the direction of the least resistance. 
Kirov G, Benchev R., Stoyanov St. ...

Deep cervical abscesses and phlegmonas usually develop after infections of tonsillar or dental origin, but there are cases when the primary site cannot be found. The most common ways of spread are the lymphogenic - to the cervical lymph nodes and hematogenic. When the local immunity is insufficient for bacterial eradication, starts suppuration and abscess is formed.

Deep neck abscesses and phlegmonas are potentially life threatening, due to the danger for airway obstruction, development of descending mediastinitis and sepsis. Even in cases with implication of the contemporary therapeutic modalities, the mortality in descending mediastinitis reaches $40 \%$. Its fast development can lead to late diagnosis with poorer prognosis.

Our observations showed that sometimes common tonsillar, dental or ear infections can have an unexpected course and cause severe life threatening complications. This demands careful examination and close follow up of the patients.

\section{CONCLUSION}

In conclusion, we want to underline the importance of early diagnosis and urgent surgical treatment of the neck infections, in combination with suitable antibiotic, covering aerobe and anaerobe microorganisms. The good coordination between otorhinolaryngologist, surgeon, anesthesiologist and microbiologist is of utmost importance. The best prophylaxis of deep neck abscesses is the early and adequate treatment of the primary infection sites.

\section{REFERENCE}

1. Pavlov V., R. Benchev. Descending mediastinitis as complication of neck abscesses and phlegmonas. Otorhinolaryngology 1997; 2-3: 47-51.

2. Pavlov V., R. Benchev. Some aspects of the treatment of neck abscesses and phlegmonas. Otorhinolaryngology; 1998; 2: 38-46.

3. Amin a. et al. Retropharyngeal and mediastinal abscess: resolution by cervical drainage. Otolaryngol Head Neck Surg 1992; 107: 2999.

4. Beasty D., Amadee R. Deep neck infections. $J$. LA State Med Soc 1995; 147: 181-184.

5. Brunelli A. et al. Descending necrotizing mediastinitis. Arch Otolaryngol Head Neck Surg 1996; 112: 1326-1329.

6. Estera A. et al. Descending necrotizing mediastinitis. Surg Gynecol Obstetr 1983; 157: 545-552.

7. Johnson J. Deep neck abscesses. Chapter 71 in Operative otolaryngology, head and neck surgery 1997, Editor E. Myers, W.B. Saunders company.

8. Spodaryk et al. Mastoiritis - a forgotten disease? Przegl Lek 1992; 49: 403-405.

9. Wheaty M., M. Stirling, M. Kirsh. Descending necrotizing mediastinitis: Trans-cervical drainage is not enough. Ann Thoracic Surg 1990;49: 780-784

10. Venezio F., T. Naidich, S. Schulmann. Complications of mastoiditis with special emphasis on venous sinus thrombosis. J Pediatr 1982; 101: 509-513. 